\title{
Volker HUGENSCHÜTT (1997): Bioindikationsanalyse von Uferzonationskomplexen der Spinnen- und Laufkäfer- gemeinschaften (Arach.: Araneae, Col.: Carabidae) an Fließgewässern im Drachenfelser Ländchen. Dissertation, Rheinische Friedrich-Wilhelms-Universität Bonn, $350 \mathrm{~S}$.
}

In derneu vorgelegten Arbeit wird die Eignung von Carabiden und epigäischen Araneae als anwendungsorientierte Bioindikatoren für unterschiedliche Uferstrukturausprägungen kleiner Fließgewässer überprüft. An fünf im Natürlichkeitsgrad differierenden Uferstandorten wurden die Zonationszönosekonstellationen der beiden Arthropodengruppen untersucht. Über einen Zeitraum von 5 Jahren wurden die Laufkäfer und begleitend über 3 Jahre die Webspinnen ohne Berücksichtigung des Winteraspektes mit Einsatz der Bodenfallenmethode nach BARBER erfaßt. Um gemäß der Fragestellung neben der Repräsentanzanalyse der Einzelflächen auch den Uferzonenkomplex des Bachsystems beurteilen zu können, wurden die Fallen ausgehend von der amphibischen bis in die terrestrische bzw. agrarische Uferregion hinein in senkrechtzum Bach verlaufenden Transekten angelegt. Um die unteren Dimensionsgrenzen der räumlichen Größenordnungen der Uferzonationszönosen besser erreichen zu können, wurden zusätzlich Kurztransekte in Form von drei Fallen auf einer Transektlänge von nur $2 \mathrm{~m}$ installiert. Die Standorte wurden vegetationskundlich umfassend beschrieben und nach ihren Mikroklimaverhältnissen differenziert.

Der Gesamtfang der Carabidae beträgt 10'134 Individuen, die innerhalb von 18 Unterfamilien 88 Arten umfassen, während aus 13'439 gefangenen Webspinnen 139 Arten aus 22 Familien determinierbar waren.

Den Zönose-Analysen wurde eine Reihe statistischer und autökologischtypologischer Parameter zugrunde gelegt. Die umfassend vorgenommene, detailierte Charakterisierung der Arten nach Biotop-Präferenztypen läßt sich aus Gründen der Übersicht und Vergleichbarkeit für Carabiden und Webspinnen auf fünf einheitlich definierte Ökotypen einschränken. Um in der vergleichenden Beurteilung der Probeflächen die regionale Stenökie der Arten berücksichtigen zu können und einen lokalen Bezugspunkt zu gewährleisten, wurde eine richtlinienbestimmende, naturnahe Waldbachaue als "Eichstandort" für die zu untersuchenden Ist- und Sollwertbedingungen deklariert. 
Für die indikative Beurteilung der Standortqualität auf Artniveau konnte ein neues Artwertkonzept als eine für Bachuferauen anwendbare, regional unabhängige Zustands-Wertigkeits-Relation (ZWR) entwickelt werden.

Die für Araneae und Carabiden ausgewählten Indikationsparameter und Zönosekenndaten ermöglichen den Nachweis und eine eindeutige Differenzierung der sowohl standortbezogenen als auch gesamtzonal ausgeprägten Uferzonationszönosen. Über die Verteilung der Ökotypen ist für die Webspinnen eine im Vergleich zu den Carabiden geringere uferzonendifferenzierende Fähigkeit festzustellen. Innerhalb der Kurztransekte werden die extrem schmalräumig abgefaßten, unmittelbaren Uferzonen von der relativen Habitatbindung der Araneae daher nicht in der Schärferelation getrennt dargestellt, wie dies die entsprechenden Carabidenökotypen zu leisten vermögen. Die prägnante zönologische Zonendifferenzierung innerhalb der nur 2 m langen Kurztransekte läßt eine noch kleiner dimensionierte zonale Graduierung der Bachuferlebensgemeinschaften erwarten. Der Gesamtindividuenanteil der obligat hygrobionten Spinnenarten liegt um fast $16 \%$ unter dem der Laufkäfer. Die erhebliche Störgröße der Hochwasserereignisse verhindert die Genese einer spezifischen autochthon-ripicolen Uferspinnenzönose. Den höchsten zonalen Stenökiegrad prägen die obligat hygrobionten Arten aus, die sich - auch standortqualitätsübergreifend - in den amphibisch beeinflußten, oft auf wenige Zentimeter eingeschränkten Uferzonen konzentrieren. In der amphibisch-terrestrischen Übergangszone des Eichstandortes findet sich eine doppelt so hohe Dominanz an feuchtepräferenten Carabidenarten wie in den anderen, pessimierten Probeflächen. Der Artwert unterstreicht den hohen ökologischen Stellenwert der amphibischen Litoraea-Zone mit den sowohl für Carabiden als auch für Bodenspinnen standortintern jeweils höchsten Punktzahlen.

Die verschiedenen Bindungs- und Anpassungsmechanismen der Ufercarabiden an die aquadynamische Wasser-Land-Kontaktzone werden diskutiert. Die Ökotypendominanz-Konstellation und die Dominanzstrukturkurve erweisen sich als die aussagekräftigsten ökologischen Vergleichsgrößen, um die Auswirkungen der nachteilig entwickelten Uferstandorte auf die Saumzönosen abzuschätzen. Die durch die Uferstrukturen bedingten Variationen der Körpergrößen-Biomasse-Relation und die Berechnung des Spinnenprädationsvolumens ermöglichen eine standortqualifizierende Aussage über das Sukzessionsstadium, das Ressourcenangebot und die Stabilität des Uferbiotops. Eine fehlende Uferbeschattung und zu schmal angelegte Gehölzgürtel führen gleichermaßen für Spinnen und Laufkäfer zu einer untypisch positiven Dominanzreaktion allochthoner Faunenelemente gegenüber zurückweichenden indigenen Standortspezialisten. Eurypotenten 
Feldarten wird durch das wachsende Randzonen-Kernflächen-Verhältnis und die fehlende Barrierewirkung einer Waldklimaschranke ein Durchsiedeln potentieller Uferauwaldflächen erleichtert.

Der nachgewiesene "Faunenschnitt" zwischen den hygrobionten und silvicolen Laufkäfer- und Webspinnenarten der Ufergehölzzone und den Feldarten der angrenzenden Ackerflächen schließt eine Wechselwirkung zwischen diesen Biotopen weitgehend aus. Die gegenüber Hecken doppelt so hohen Artenzahlen der untersuchten Ufergehölze sowie die geringen Zonenarten-Identitäten dokumentieren die Existenz von ufersauminternen Grenzlinien mit deutlichen Edge-Effekten. Selbst stark reduzierte, unbestockte Ufersäume bieten durch das vom Gewässer aufrecht gehaltene Mindestfeuchtigkeitsmilieu eine strikt eingehaltene Leitlinie für eurytope Feuchtwaldarten, um in für sie ungünstige Landschaftsformationen zu immigrieren. Erst die auf über $15 \mathrm{~m}$ ausgeweiteten Ufergehölzstreifen sichern in ihrer waldanalogen Mikroklima- und Habitatstruktur einer Reihe von stenotopen Waldspinnen ein reproduktives Vorkommen in strukturarmen Kulturlandschaften. Der Gesamtfang der Agrarzone beruht zu 69\% auf dem Vorkommen der beiden Feld-Linyphiiden Erigone atra und Oedothorax apicatus. Die relativ starke Assoziation zwischen einer angrenzenden Feuchtgrünlandsynusie und hygrobionten Uferrandarten unterstützt die Naturschutzforderung bachanliegende Agrarflächen höchstens als extensives Grünland zu nutzen.

Die Bioindikationsfähigkeit des Untersuchungsansatzes und die Anwendung des Artwertes werden literaturvergleichend diskutiert. Die aus den Ergebnissen ableitbaren Konsequenzen für eine Biotopoptimierung und entsprechende Maßnahmen werden vorgestellt und diskutiert.

Volker HUGENSCHÜTT, Bismarckstrasse 33, D-53113 Bonn 